\title{
Exercise Overcomes Muscle Weakness Following on Trauma and Critical Illness
}

\begin{abstract}
Injuries related to trauma are often seen in South African intensive care units. Systemic inflammation and the development of sepsis lead to prolonged intensive care unit and hospital stay. The effects of critical illness and immobility on the human body are not always wellunderstood. This review article explains the pathogenesis of muscle weakness due to inflammation and identifies the role of exercise prescription in counteracting impairments that may be identified in survivors of trauma during and after hospital stay.
\end{abstract}

\section{Van Aswegen H, PhD'; Myezwa $\mathrm{H}$, MSc Physiotherapy ${ }^{1}$}

1 Physiotherapy Department, School of Therapeutic Sciences, University of the Witwatersrand.

KEYWORDS: TRAUMA, INFLAMMATION, MUSCLE WEAKNESS, EXERCISE.

\section{INTRODUCTION}

South Africa has a high incidence of violence and death due to high levels of interpersonal violence and motor vehicle accidents. Norman and colleagues (2007) reported a homicide rate in South Africa that was seven to nine times greater than global reported rates. The authors also reported a mortality rate related to road traffic accidents in South Africa of double the global rate. Thomson (2003) stated that trauma kills young people in the prime of their economically productive lives. Trauma is often referred to as the 'hidden epidemic' as it gets much less media attention than the human immunodeficiency virus/acquired immunodeficiency syndrome (HIV/AIDS) pandemic but has an equally high mortality rate (Thomson 2003). Survivors of trauma often present with serious injuries and illness that necessitate

\section{Correspondence to:}

Heleen van Aswegen

Physiotherapy Department,

School of Therapeutic Sciences,

University of the Witwatersrand,

7 York Road, Parktown

Johannesburg 2050

Tel: (011) 717-7302 (w)

Fax: (011) 717-3719 (w)

Email: helena.vanaswegen@wits.ac.za admission to the intensive care unit (ICU). Severity of illness determines the length of ICU and hospital stay. The 2002 Brussels Roundtable Discussions among intensive care practitioners concluded that survivors of intensive care suffered from poor functional capabilities, decreased quality of life (QOL), and decreased rate of return to work and placed an increased burden and stress on families and informal caregivers. These factors led to increased economic costs for the patient, families and the society (Angus \& Carlet 2003).

The effects of immobility and critical illness on the human body (short and long-term) are not always well-understood. The aims of this review article are to a) provide information to physiotherapists on the human body's response to trauma; b) explain the pathogenesis of muscle weakness due to inflammation and the resulting impairments that survivors present with and c) identify the role of exercise prescription in counteracting these impairments both during and after hospital stay.

\section{TRAUMA AND THE HUMAN BODY}

Figure 1 outlines the responses of body systems to trauma. Local and systemic responses are activated by various factors such as injury, surgery, burns, dehydration, sepsis and acute medical illness.
Acute injury/trauma leads to loss of lean body and skeletal mass and precedes the process of recovery and wound repair. Inflammation, as a result of injury, consists of humoral [triggering of coagulation and fibrinolytic cascades as well as kinin (blood protein that acts as vasodilator during inflammation i.e. bradykinin)] and cellular (activation of leukocytes and endothelial cells) responses. Exacerbation of the inflammatory response can lead to the development of a systemic inflammatory response syndrome (SIRS) due to an imbalance in the production of proinflammatory cytokines (Hietbrink et al 2006). Anti-inflammatory cytokines are released to restore this imbalance but over activation leads to either a compensatory anti-inflammatory response or a mixed antagonist response (Hietbrink et al 2006; Osuchowski et al 2006). The patient's immune system is in disarray and the patient becomes very susceptible to infection which might result in septic syndrome and ultimately multiple organ dysfunction (MOD) (Hietbrink et al 2006; Osuchowski et al 2006).

The initial response to trauma is to access energy from endogenous fat oxidation. Fat provides most of the body's energy requirements during the period in which no food is ingested. Fat is mobilized from fat stores and converted 


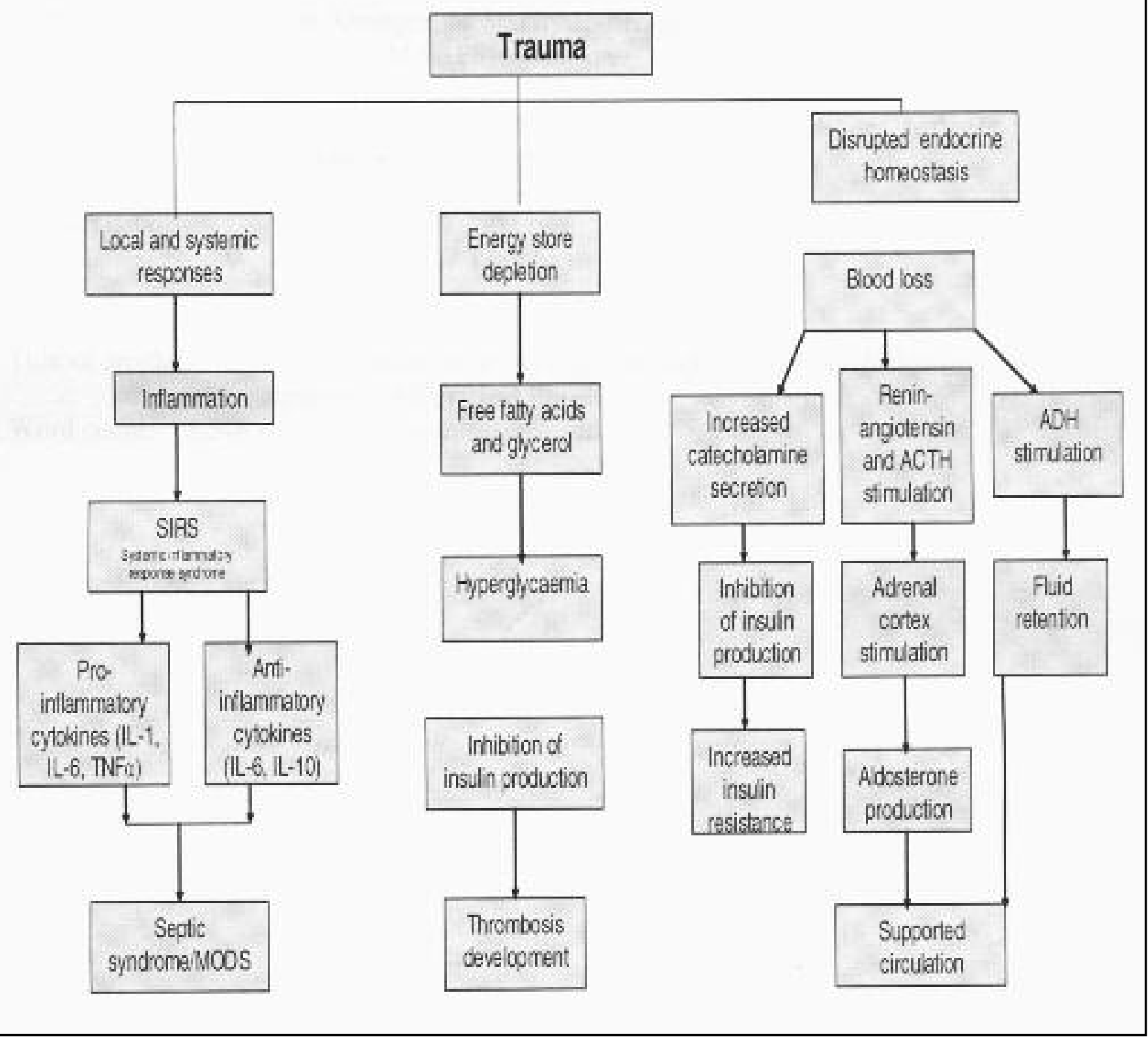

to free fatty acids and glycerol. These are then circulated to tissues such as the large muscles which can burn the fatty acids directly. Blood sugar starts to rise slowly and insulin production from the pancreas is inhibited (Boffard 2003; Winkler \& Manchester 1996). A metaanalysis of randomised controlled trials (RCT) on insulin therapy administration during critical illness showed that hyperglycemia is common in patients with critical illness and also may play a role in the activation of the tissue factor pathway of coagulation (Pittas, Siegel \& Lau 2004). This may lead to the development of acute thrombosis. The authors concluded that insulin therapy and tight glucose control had a beneficial effect on short-term morbidity and mortality (Pittas, Siegel \& Lau 2004). Hyperglycaemia may contribute to the patient's mortality rate. Gale and coworkers recently published results from a retrospective study that was performed on all trauma patients in their urban hospital (Gale et al 2007). They found that poor glycaemic control was associated with increased morbidity and mortality in critically ill trauma patients and was more pronounced in non-diabetic patients. They also found a greater prevalence of urinary tract infections and complications in non-diabetic trauma patients with poor glycaemic control. These results have prompted many South African trauma surgeons to treat their patients, regardless of admission diagnosis, with insulin therapy to maintain glucose levels within the normal range during their ICU stay.

Trauma also disrupts endocrine homeostasis. Blood loss is the most important stimulant of aldosterone. This occurs via the renin-angiotensin system and adrenocorticotropic hormone (ACTH) both of which stimulate the adrenal cortex to produce aldosterone. Aldosterone supports circulation through decreased excretion of sodium bicarbonate through the kidneys. Blood loss also stimulates the production of vasopressin/antidiuretic hormone (ADH) from the posterior pituitary, with consequent fluid retention and vasoconstriction of the large blood vessels. Increased catecholamine secretion is the main endocrine response to trauma. These catecholamines, especially epinephrine, inhibit insulin production and increase insulin resistance. This causes an initial, slight elevation of the blood sugar and a gradual rise in glucagon levels (Boffard 2003; Davis 2001). 
Trauma patients often develop circulatory shock due to severe blood loss, myocardial contusion, cardiac tamponade, tension pneumothorax or spinal injury. Shock leads to the development of various degrees of cellular damage, depletion of cellular energy stores (ATP), myocardial dysfunction, oliguria (decrease in urine output) and splanchnic ischaemia due to hypoperfusion. Hyperventilation occurs due to lactic acidosis and reduced pulmonary blood flow that leads to ventilation-perfusion mismatch and shunting (Schiller \& Anderson 2001; Tatsumi et al 2000). SIRS results in capillary leak associated with the development of the acute respiratory distress syndrome (ARDS) manifested by progressive posterior atelectasis. Respiratory muscle fatigue from muscle hypoperfusion and increased energy expenditure may lead to respiratory failure (Schiller \& Anderson 2001; Skowronski 1998). The patient's prognosis depends on the severity of the injury, age and pre-existing illnesses. Survival depends on maintenance of oxygen delivery and effective surgical intervention (Schiller \& Anderson 2001; Skowronski 1998).

\section{THE INFLAMMATORY PROCESS}

The human body is protected from a wide range of micro-organisms through the immune system mediated through antigen presenting cells and inflammatory cells. These cells produce proinflammatory and anti-inflammatory cytokines, lymphokines and eicosanoids that mediate the response to an inflammatory stimulus (infective or not). Pro-inflammatory mediators include interleukins (IL) 1 and 6, tumour necrosis factor-alpha (TNF-a), prostaglandins, leukotrienes and reactive oxygen species (ROS) (Grimble 1999; Mukhopadhyay, Hoidal \& Mukherjee 2006). Anti-inflammatory mediators cause immunosuppression and include IL-4, IL-10, IL-13, soluble TNF receptors and IL-1 receptor antagonist. Cytokines such as the IL and TNF-a are released into the circulation by macrophages and monocytes in inflamed tissues. Cytokines are proteins that are produced by white blood cells. IL facilitates inflammation and promotes white cell proliferation and infiltration. IL can be classified as pro-inflammatory, anti-inflammatory or have both properties (Winkelman 2004). Research is frequently conducted on the role of IL-1, IL-6 and IL-10 in critical illness (Winkelman 2004).

\section{Interleukin-1}

IL-1 can be categorised as IL-1A and IL-1B. Both bind to receptors on the cell membrane (Winkelman 2004) and small amounts can elicit an inflammatory response throughout the body. IL-1 is therefore associated with the development of SIRS and causes clinical reactions such as fever, malaise and loss of appetite and sleep (Winkelman 2004). IL-1 promotes the adhesion of leukocytes to the endothelium resulting in capillary leak, stimulates the production of white blood cells from bone marrow and promotes prostaglandin production. Both IL-1A and IL-1B are toxic to muscle tissue (Winkelman 2004). IL-1A influences insulin-growth factor and this leads to muscle protein breakdown. IL-1B is known to be involved with apoptosis (planned cell death with subsequent phagocytosis by other cells) and contributes to the reduction of muscle mass (Winkelman 2004).

\section{Interleukin-6}

IL-6 is released from T-cells, mast cells, epithelial cells, pulmonary epithelial cells and myocytes. It stimulates the activation of neutrophils and natural killer cells with further release of IL-1 and TNF- $\alpha$. It enhances B-cell production as well as antibody production by B-cells (Winkelman 2004) and as inflammation becomes chronic it slows the production of IL- 1 and TNF- $\alpha$ and promotes the release of anti-inflammatory mediators. Research has shown that persistently high levels of circulating IL-6 are associated with an increased risk of mortality in patients suffering from sepsis, trauma or burns (Winkelman 2004). IL-6 enhances the infiltration of myocytes with prostaglandins, which leads to breakdown of protein, degeneration of myocytes and muscle atrophy. IL-6 has been associated with maintenance of homeostasis during exercise through the regulation of glucose metabolism by skeletal muscles (Winkelman 2004).

\section{Interleukin-10}

IL-10 is an anti-inflammatory cytokine that inhibits the production of proinflammatory cytokines by macrophages or monocytes. Low levels of IL-10 in the ICU patient can be associated with excessive inflammation and muscle damage and lead to the development of SIRS. High levels of IL-10 may provide protection against inflammatory myopathy. Myocyte damage as a result of disease progression is best controlled by maintaining a balance between proinflammatory cytokines (IL-1 and IL-6) and anti-inflammatory cytokines (IL-10) (Pretorius 2003; Winkelman 2004).

\section{Tumour Necrosis Factor-Alpha}

TNF- $\alpha$ is released into the circulation during the early phase of injury or infection. TNF- $\alpha$ produces similar metabolic and physiologic actions to IL-1 (Grimble 1999; Mukhopadhyay, Hoidal \& Mukherjee 2006).

\section{Reactive Oxidant Mediated Injury}

Recent developments in the medical literature propose that systemic oxidative stress plays a role in the development and manifestation of critical illness. Oxidative stress has been defined as "a condition in which accumulation of free radicals or the inability of antioxidants to counter the accumulation of free radicals creates an imbalance between production of ROS and protection of antioxidants" (Goodyear-Bruch \& Pierce 2002, pp 545). In health small amounts of ROS are essential to life, forming a major component of our intrinsic defence systems in the form of phagocyte derived hydrogen peroxide and hypochlorous acid. These and others that are produced as by-products of normal metabolism, (such as purine and arachidonic acid metabolism) are neutralized by the major endogenous anti-oxidants glutathione, catalase, superoxide dismutase and albumin and exogenous antioxidants such as vitamin $\mathrm{C}$, vitamin $\mathrm{E}$, $\beta$ carotene and selenium. However in illness, increased generation of ROS results in the consumption of antioxidants and an oxidant/anti-oxidant imbalance ensues (Cuzzocrea, Thiemerman \& Salvemini 2004; Salvemini \& Cuzzocrea 2002). ROS themselves 
stimulate the inflammatory response by increasing the release of cytokines and thus activate the inflammatory cascades (Goodyear-Bruch \& Pierce 2002). Loss of control of the production of ROS in SIRS and sepsis leads to direct cellular injury through oxidative damage to cellular proteins, interstitial cell structures and the destruction of cell membranes. This sequence of events may result in multiple organ failure (Abilés et al 2006; Goodyear-Bruch \& Pierce 2002; Zhang, Slutsky \& Vincent 2000). Ely and colleagues stated that MOD syndrome occurring as a result of sepsis is the most common cause of mortality among critically ill patients without coronary complications (Ely, Kleinpell \& Goyette 2003).

\section{Pathogenesis of Muscle Weakness}

TNF-a increases the production of catabolic cytokines (IL-1 and IL-6) and causes anorexia (Jackman \& Kandarian 2004; Reid \& Li 2001; Winkelman 2004). This catabolic effect leads to the disruption of myogenesis (formation of muscle tissue) (Andreoli et al 1993). It causes muscle breakdown in inflammatory diseases such as cancer, chronic obstructive pulmonary disease (COPD), AIDS and sepsis, which contributes to weakness, fatigue and limited mobility (Reid \& Li 2001). TNF- $\alpha$ binds to Type 1 TNF- $\alpha$ receptors leading to an increased production of ROS from mitochondrial electron transport chains in skeletal muscle (Reid \& Li 2001). Nuclear factor- $\kappa \mathrm{B}$ is activated causing increased activity of the ubiquitin/proteasome pathway that accelerates degradation of the bulk of all intracellular proteins, especially myofibrillary protein and promotes muscle weakness (Jackman \& Kandarian 2004; Reid \& Li 2001; Winkelman 2004). Reid compared the effect of TNF- $\alpha$ on the contractile function of a murine diaphragm and the flexor digitorum brevis muscle. They found that TNF- $\alpha$ induced contractile dysfunction on both muscles through its effect on the myofilaments. They stated that TNF- $\alpha$ promotes the production of ROS and nitric oxide derivatives (Reid, Lännergren \& Westerblad 2002).

Jackman and Kandarian postulated that TNF-a and other cytokines act as triggers for muscle wasting due to the effect of cachexia on the body as a whole. They stated that ROS contribute to the cytokine-induced muscle wasting (Jackman \& Kandarian 2004). Lanone and colleagues (2000) investigated the effects of sepsis on muscle contractile failure in a group of 16 patients. Biopsies from the rectus abdominis muscles were compared with biopsies taken from patients without sepsis that underwent elective surgery. The authors found a marked decrease in the contractile force of the rectus abdominis muscles of patients with sepsis compared to the controls. They ascribed this finding to the fact that high levels of nitric oxide synthase were present in the muscle samples taken from patients with sepsis. Nitric oxide synthase leads to the production of nitric oxide which interacts with ROS to form peroxynitrites that directly attack contractile proteins (Lanone et al 2000). Eikermann and colleagues (2006) performed a similar experiment on patients that had survived sepsis and MOD syndrome. They investigated the effect of sepsis and MOD syndrome on the strength and fatigue of the adductor pollicis (AP) muscle. They found that AP muscle force was significantly decreased (by 30\%) compared with controls but that no fatigability was observed. They concluded that the decrease in muscle force was due to a sepsis-induced myopathy as the compound muscle action potential and nerve conduction velocity was normal, implying no neural injury (Eikermann et al 2006).

\section{Prolonged immobilisation and its effect on the human body}

The physical deconditioning that result from prolonged bed rest is not new to medical science. Hippocrates described loss of muscle strength and reduced exercise performance following prolonged bed rest and inactivity in the earliest medical journals. Bed rest is generally an unavoidable consequence of critical illness (Convertino, Bloomfield \& Greenleaf 1997; Winkelman 2004). The adverse effects of prolonged bed rest in the supine position include loss of hydrostatic pressure in the cardiovascular system below the level of the heart; elimination of longitudinal com- pressive forces on the lower limbs and spine with reduced muscle force exerted on all weight bearing bones; a significant decrease in total energy utilization and detrimental effects on the musculoskeletal system with regard to muscle power, endurance and size of muscle fibres (Convertino, Bloomfield \& Greenleaf 1997). This results in weakness and easy fatigability of the diaphragm and skeletal muscles (Winkelman 2004). Disuse atrophy is exacerbated by the effects of steroids and neuromuscular blocking agents on the muscle fibres (Bolton 1996), malnutrition and the inflammatory response and prolonged periods of continuous mandatory ventilation (Winkelman 2004).

\section{The Effect of Exercise on Serum Levels of Pro- And Anti-Inflammatory Cytokines}

IL-6 generally increases within the muscle in response to exercise. The IL-6 cytokine has a complex action and has been referred to as 'good guy or bad guy' (Pederson et al 2007). An important finding in healthy subjects regarding IL-6 is that during exercise its function changes from pro-inflammatory to antiinflammatory. IL-6 inhibits TNF alpha production and enhances insulin uptake (Pederson et al 2007).

Winkelman suggests that exercise can influence the serum levels of pro- and anti-inflammatory cytokines by restoring the imbalance that exists during critical illness and suggests that exhaustive exercise has the potential to increase the levels of IL-6, IL-10 and TNF- $\alpha$ dramatically (Winkelman 2004). Similar effects of exhaustive exercise on the release of ROS were reported by Kalokerinos (2005). Moderate exercise slows the atrophic changes that occur due to disuse by improving blood flow to muscles and joints. Therapeutic activities that are performed by physiotherapists and nursing staff on patients in the ICU include positional changes from supine to side lying, passive joint range of motion (ROM) movements, sitting up over the side of the bed and transferring from bed to chair. Winkelman and colleagues studied the effect of low-level activity (position changes and passive ROM movements) on the levels of IL-6 and IL-10 in a group of 10 
patients with prolonged MV. They reported that activity did not exacerbate the levels of circulating IL-6 and IL-10 and that low-level activity in the ICU may play a role in restoring the balance in cytokine activity. The small sample size however restricted the interpretation of their findings (Winkelman et al 2007). There exists no clinical research data regarding the appropriate intensity, frequency or mode of exercise in the acutely ill patient. Stiller and Phillips (2003) suggest that exercise intensity, frequency and mode be adjusted to each individual patients' medical condition and level of debilitation. These authors recommend starting with low level activities (bed exercises and lifting of the patient into a chair) that are gradually progressed to basic mobility (standing and walking) (Stiller \& Phillips 2003).

In summary, inflammation inhibits the production of protein and the protein content of muscle tissue. This leads to loss of muscle mass and power. Inactivity, in the presence of inflammation, may contribute to the destruction of muscle cells. IL-1, IL- 6 and TNF- $\alpha$ are involved in muscle breakdown during an inflammatory reaction whereas IL-10 inhibits the actions of the abovementioned cytokines. Low-level exercise may slow the atrophic changes that occur due to disuse by improving blood flow to muscles and joints provided the patient is not too sedated to participate in these exercises.

\section{Exercise and its potential role inreha- bilitation}

It is well known that physical fitness plays a key role in a person's ability to perform activities of daily living (ADL). Physical fitness also influences their ability to participate in sport and exercise and aids in improving QOL (Blair, LaMonte \& Nichaman 2004), through the known effects on the musculoskeletal, respiratory and cardiovascular systems (Warburton, Nicol \& Bredin 2006). As discussed above, prolonged bed rest results in muscle weakness, poor muscle endurance and rapid loss of calcium from bone (Conroy \& Earle 2000; Convertino, Bloomfield \& Greenleaf 1997). Other beneficial effects of exercise are increased muscle mass and strength due to a) improved neural control, b) increased muscle cross-sectional area, c) new bone formation stimulated through weight-bearing exercises, d) increased synthesis of high-density lipoproteins and improved insulin sensitivity (Taylor, Bell \& Lough 2002). Muscles develop new capillaries which increase the oxygen extraction ratio. More skeletal muscle cell mitochondria are produced as a result of aerobic exercise and maximal oxygen uptake improves as physical fitness improves (Williams 2000). As maximal oxygen uptake increases, less energy is utilized to perform ADL and health-related QOL improves.

Rehabilitation programmes that consist of aerobic and resistance exercises are commonly used in the chronic cardiac and pulmonary disease populations with encouraging results (AACVPR 2004). Jones and co-workers (2003) are the only researchers to date to test the effectiveness of a rehabilitation programme on the recovery of survivors of critical illness in a RCT. They compiled a 93-page rehabilitation package that consisted of a self-directed exercise programme, diagrams and illustrations. The manual also contained advice on a wide range of psychological, psychosocial and physical problems that the ICU survivor could expect to encounter after they had recovered from critical illness. Subjects ( $n=69)$ and controls $(n=57)$ were contacted telephonically three times per week after discharge to monitor progress however subjects in the experimental group were also encouraged to use the self-help rehabilitation manual. All subjects were tested at follow-up clinics at eight weeks and six months. The authors found that the SF-36 physical function score for subjects in the experimental group was closer to normal and significantly different from those in the control group (Jones et al 2003).

There are few studies that investigated the effects of critical illness on recovery of exercise capacity in survivors over the first six months to one year after discharge. Herridge and colleagues (2003) evaluated the outcome of 109 adult survivors of ARDS over a oneyear period following discharge. These patients had a median injury severity score (APACHE II) of 23, median ICU length of stay (LOS) of 25 days and a median hospital LOS of 48 days. They found that survivors of ARDS still had limitations in functional abilities one year following discharge. They ascribed this to the severity of muscle wasting that took place in hospital and the resultant weakness that persisted after discharge. They emphasized that extrapulmonary disease was the main cause of limitations in exercise capacity in these subjects (Herridge et al 2003). Hui and colleagues (2005) examined the impact of severe acute respiratory syndrome (SARS) on the recovery of 110 survivors at three and six months following discharge from ICU. A mean hospital LOS of 32.4 days was reported. The authors reported that survivors of SARS had significantly lower levels of functional ability and health status when compared to the normal population at six months. They ascribed these findings to muscle deconditioning and steroidinduced myopathy (Hui et al 2005).

Due to the deconditioning that patients present with after prolonged bed rest, the only form of aerobic exercise they perform comfortably and conveniently is walking (Warburton, Nicol \& Bredin 2006). Walking involves the large muscle groups and has few, if any, side effects and has potential benefits of aerobic exercise (Morris \& Hardman 1997). Stiller and Phillips (2003) suggests 50 - 60\% of maximal $\mathrm{HR}$ as a baseline level of intensity for increasing patient fitness after discharge. The introduction of resistance and flexibility exercises for survivors of critical illness for upper and lower limb as well as trunk muscles should be done gradually in order to allow for wound healing to take place.

\section{CONCLUSION}

There is a dearth of literature on the recovery of muscle strength and exercise capacity in survivors of critical illness over the first 12 months after discharge from the hospital. Physiotherapists need to be aware of the pathogenesis of muscle weakness during critical illness and how their interventions (exercise) influence patient outcome in and after ICU. 


\section{REFERENCES}

Abilés J, De La Cruz AP, Castaño J, RodriguezElvira M, Aguayo E, Moreno-Torres R, Llopis J, Aranda $P$, Argüelles S, Ayala A, De La Quintana AM, Planells EM 2006 0xidative stress is increased in critically ill patients according to antioxidant vitamins intake, independent of severity: a cohort study. Critical Care 10 (5): R146.

American Association of Cardiovascular and Pulmonary Rehabilitation 2004 Guidelines for Pulmonary Rehabilitation Programmes, 3rd edn, Human Kinetics Publishers, USA.

Andreoli TE, Bennett JC, Carpenter CCJ, Plum F, Smith LH Jr 1993 Cecil Essentials of Medicine, 3rd edn, WB Saunders Company, Pennsylvania, pp.639 - 641 .

Angus DC, Carlet $\mathrm{J}$ on behalf of the 2002 Brussels Roundtable Participants 2003 Surviving intensive care: a report from the 2002 Brussels Roundtable. Intensive Care Medicine 29: 368 - 377.

Blair SN, LaMonte MJ, Nichaman MZ 2004 The evolution of physical activity recommendations: how much is enough? The American Journal of Clinical Nutrition 79 (supplement): 913S - 920 S.

Boffard KD 2003 Metabolic response to trauma. In: CJ Mieny, U Mennen (eds) Principles of Surgical Patient Care, 2nd edn, New Africa Books, Claremont, pp. 170 - 178.

Conroy B, Earle RW 2000 Bone, muscle and connective tissue adaptations to physical activity. In: TR Baechle, RW Earle (eds) Essentials of Strength Training and Conditioning: National Strength and Conditioning Association, 2nd edn, Human Kinetics, Champaign, Illinois, pp. $57-72$.

Convertino A, Bloomfield SA, Greenleaf JE 1997 An overview of the issues: physiological effects of bed rest and restricted physical activity. Medical Science and Sport Exercise 29 (2): $187-190$.

Cuzzocrea S, Thiemermann C, Salvemini D 2004 Potential therapeutic effect of antioxidant therapy in shock and inflammation. Current Medicinal Chemistry 11 (9): 1147 - 1162.

Davis KD 2001 Thyroid and adrenal disorders. In: PN Lanken (ed) The Intensive Care Unit Manual, WB Saunders Company, Pennsylvania, pp. 931 - 947.
Eikermann M, Koch G, Gerwig M, Ochterbeck C, Beiderlinden $M$, Koeppen S, Neuhäuser M, Peters J 2006 Muscle force and fatigue in patients with sepsis and multiorgan failure. Intensive Care Medicine 32: 251 - 259.

Ely EW, Kleinpell RM, Goyette RE 2003 Advances in the understanding of clinical manifestations and therapy of severe sepsis: an update for critical care nurses. American Journal of Critical Care 12: 120 - 133.

Gale SC, Sicoutris C, Reilly PM, Schwab CW, Gracias VH 2007 Poor glycemic control is associated with increased mortality in critically ill trauma patients. The American Surgeon 73 (5): 454 - 460.

Goodyear-Bruch C, Pierce JD 2002 Oxidative stress in critically ill patients. American Journal of Critical Care 11 (6): 543 - 551.

Grimble RF 1999 Nutritional influences on inflammation. In: BR Bistrian, JA Walker-Smith (eds) Nestle Nutrition Workshop Series Clinical and Performance Programme, vol 2, Karger AG, Basel, pp. 63 - 81.

Harman E 2000 The biomechanics of resistance exercise. In: TR Baechle, RW Earle (eds) Essentials of Strength Training and Conditioning: National Strength and Conditioning Association, 2nd edn, Human Kinetics, Champaign, Illinois, pp. 25 - 56.

Herridge MS, Cheung AM, Tansey CM, MatteMartyn A, Diaz-Granados N, Al-Saidi F, Cooper AB, Guest CB, Mazer CD, Mehta S, Stewart TE, Barr A, Cook D, Slutsky AS 2003 One-year outcomes in survivors of the acute respiratory distress syndrome. New England Journal of Medicine 348: 683 - 693.

Hietbrink F, Koenderman L, Rijkers GT, Leenen LPH 2006 Trauma: the role of the innate immune system. World Journal of Emergency Surgery 1: 1 - 15.

Hui DS, Joynt GM, Wong KT, Gomersall CD, Li TS, Antonio G, Ko FW, Chan MC, Chan DP, Tong MW, Rainer TH, Ahuja AT, Cockram CS, Sung JJ 2005 Impact of severe acute respiratory syndrome (SARS) on pulmonary function, functional capacity and quality of life in a cohort of survivors. Thorax 60 (5): 401 - 409.

Jackman RW, Kandarian SC 2004 The molecular basis of skeletal muscle atrophy. American Journal of Physiology: Cell Physiology 287: C834 - C843.
Jones C, Skirrow P, Griffiths RD, Humphris GH, Ingleby S, Eddleston J, Waldmann C, Gager M 2003 Rehabilitation after critical illness. Critical Care Medicine 31 (10): 2456 - 2461.

Kalokerinos A, Dettman I, Meakin C 2005 Endotoxin and vitamin C: Part 1 - Sepsis, endotoxin and vitamin C. Journal of Australasian College of Nutritional and Environmental Medicine 24 (1): 17 - 21.

Lanone S, Mebazaa A, Heymes C, Henin ID, Poderoso JJ, Panis Y, Zedda C, Billiar T, Payen D, Aubier M, Boczkowski J 2000 Muscular contractile failure in septic patients: role of the inducible nitric oxide synthase pathway. American Journal of Respiratory and Critical Care Medicine 162: 2308 - 2315.

Morris JN, Hardman AE 1997 Walking to health. Sports Medicine 23 (5): 306 - 332.

Mukhopadhyay S, Hoidal JR, Mukherjee TK 2006 Role of TNF- $\alpha$ in pulmonary pathophysiology. Respiratory Research 7: 125 - 134.

Norman R, Matzopoulos R, Groenewald P, Bradshaw D 2007 The high burden of injuries in South Africa. Bulletin of the World Health Organization 85 (9): 695 - 702.

Osuchowski MF, Welch K, Siddiqui J, Remick DG 2006 Circulating cytokine/inhibitor profiles reshape the understanding of the SIRS/CARS continuum in sepsis and predict mortality. Journal of Immunology 177: 1967 - 1974.

Pittas AG, Siegel RD, Lau J 2004 Insulin therapy for critically ill hospitalised patients: a metaanalysis of randomized controlled trials. Archives of Internal Medicine 164 (18): 2005 2011.

Pretorius JP 2003 Current concepts in sepsis and septic shock. In: CJ Mieny, U Mennen (eds) Principles of Surgical Patient Care, 2nd edn, New Africa Books, Claremont, pp. 273 - 296.

Reid MB, Lannergren J, Westerblad H 2002 Respiratory and limb muscle weakness induced by tumor necrosis factor- $\alpha$ : Involvement of muscle myofilaments. American Journal of Respiratory and Critical Care Medicine 166: 479 - 484.

Reid MB, Li YP 2001 Tumor necrosis factor- $\alpha$ and muscle wasting: a cellular perspective. Respiratory Research 2: 269 - 272. 
Salvemini D, Cuzzocrea S 2002 Oxidative stress in septic shock and disseminated intravascular coagulation. Free Radical Biology and Medicine 33 (9): 1173 - 1185.

Schiller HJ, Anderson III HL 2001 Hemorrhagic shock and other low preload states. In: PN Lanken (ed) The Intensive Care Unit Manual, WB Saunders, Pennsylvania, pp. 85 - 92.

Skowronski GA 1998 Circulatory shock. In: TE Oh (ed) Intensive Care Manual, 4th edn, Butterworth Heinemann, Bath, pp. 139 - 145.

Stiller K, Phillips A 2003 Safety aspects of mobilising acutely ill inpatients. Physiotherapy Theory and Practice 19: 239 - 257.

Tatsumi T, Matoba S, Kawahora A, Keira N, Shiraishi J, Akashi K, Kobara M, Tanaka T, Katamura M, Nakagawa C, Ohta B, Shirayama T, Takeda K, Asayama J, Fliss H, Nakagawa M 2000 Cytokine-induced nitric oxide production inhibits mitochondrial energy production and impairs contractile function in rat cardiac myocytes. Journal of the American College of Cardiology 35 (5): 1338 - 1346.

Taylor A, Bell J, Lough F 2002 Cardiac rehabilitation. In: JA Pryor, SA Prasad (eds) Physiotherapy for Respiratory and Cardiac Problems:
Adults and Paediatrics, 3rd edn, Churchill Livingstone, Edinburgh, pp. 498 - 499.

Thomson SR 2003 Trauma of the abdomen: blunt and penetrating. In: CJ Mieny U Mennen (eds) Principles of Surgical Patient Care, 2nd edn, New Africa Books, Claremont, pp. 863 -865 .

Warburton DER, Nicol CW, Bredin SSD 2006 Prescribing exercise as preventive therapy. Canadian Medical Association Journal 174 (7): $961-975$

Williams M 2000 Cardiovascular and respiratory anatomy and physiology. In: TR Baechle, RW Earle (eds) Essentials of Strength Training and Conditioning: National Strength and Conditioning Association, 2nd edn, Human Kinetics, Champaign, Illinois, pp. 123 - 131.

Winkelman C 2004 Inactivity and inflammation: Selected cytokines as biologic mediators in muscle dysfunction during critical illness. AACN Clinical Issues 15 (1): 74 - 82.

Winkelman C, Higgins PA, Chen YJK, Levine AD 2007 Cytokines in chronically critically ill patients after activity and rest. Biological Research for Nursing 8 (4): 261 - 271.
Winkler MF, Manchester S 1996 Nutritional care in metabolic stress: sepsis, trauma, burns and surgery. In: LK Mahan, S Escott-Stumps (eds) Krause's Food, Nutrition and Diet Therapy, 9th edn, WB Saunders, Pennsylvania, pp. $663-665$.

Zhang H, Slutsky AS, Vincent J-L 2000 Oxygen free radicals in ARDS, septic shock and organ dysfunction. Intensive Care Medicine 26 : 474 - 476. 